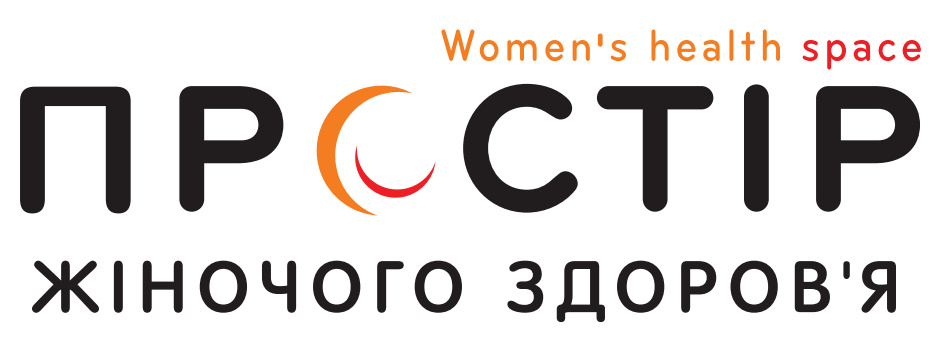

\title{
ВЛИЯНИЕ ПАТОЛОГИИ ЩИТОВИДНОЙ ЖЕЛЕЗЫ НА СТРУКТУРУ КОСТНОЙ ТКАНИ
}

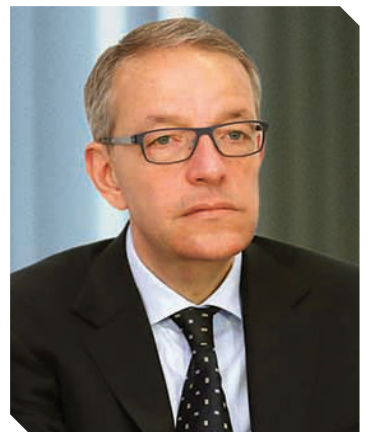

Б.Н. МАНЬКОВСКИЙ д. мед. н., профессор, член-корр. НАМН Украины, заведующий кафедрой диабетологии

Национальной медицинской академии им. П.Л. Шупика, г. Киев

Контакты:

Маньковский Борис Никитич mankovsky1964@yahoo.com
Вопрос взаимосвязи заболеваний щитовидной железы (ЩЖ) и состояния костной системы является весьма актуальным, но, к сожалению, обсуждается нечасто. Гормоны ЩЖ абсолютно необходимы для созревания и формирования костной ткани, скелета (рис. 1-4). Это очень важно с физиологической и патофизиологической точки зрения, поскольку заболевания со стороны ЩЖ клинически проявляются не только в виде классических симптомов гипо- или гипертиреоза, но также и в форме нарушений со стороны костной системы.

\section{Гunomepuos}

При снижении функции ЩЖ и выработки меньшего количества тиреоидных гормонов отмечается уменьшение активности как остеобластов, так и остеокластов. Но до сих пор остается неясным, какие именно процессы преобладают у взрослых - формирования или разрушения костной ткани, поэтому влияние гипотиреоза на костную ткань взрослых требует дальнейшего изучения. У детей этот вопрос более изучен. Установлено, что у новорожденных в антенатальном периоде гипотиреоз приводит к замедлению и нарушению формирования костной ткани, а при компенсации (адекватной заместительной терапии гормонами ЩЖ, в частности L-тироксином) эти изменения прекращаются, формирование скелета возобновляется и протекает нормально.

\section{Гunepmupeoз}

При избыточной функции ЩЖ имеется четкая взаимосвязь повышенной продукции гормонов железы (свободного тетрайодтиронина $\left(\mathrm{T}_{4}\right)$, свободного трийодтиронина $\left.\left(\mathrm{T}_{3}\right)\right)$ с развитием остеопороза. Это может наблюдаться в нескольких ситуациях: при гипертиреозе, болезни Грейвса (диффузный токсический зоб). Также эти процессы могут иметь место при субклиническом тиреотоксикозе, что особенно важно. Субклинический тиретоксикоз характеризуется сниженным уровнем тиреотропного гормона и нормальными значениями $\mathrm{T}_{4}$ и $\mathrm{T}_{3}$. В итоге данное состояние не имеет клинических проявлений, но оказывает четкое неблагоприятное влияние на структуру костной ткани, а именно приводит к остеопорозу.

Помимо этого, остеопороз может развиваться при проведении заместительной терапии гормонами ЩЖ (прежде всего L-тироксином) - кпримеру, улиц с удаленной ЩЖ по причине рака железы. Как правило, таким пациентам после хирургического вмешательства назначается супрессивная заместительная терапия, когда дозы L-тироксина превышают таковые при заместительной гормонотерапии при гипотиреозе, аутоиммунном тиреоидите Хашимото (вызвавшем гипотиреоз), после операции по поводу диффузного токсического или узлового зоба. Следовательно, после хирургического лечения рака ЩЖ заместительная гормонотерапия стремится достигнуть уровня тиреотропного гормона на нижней границе нормы (т. е. доза L-тироксина выше, чем при других гипотиреоидных состояниях), что приводит к ряду побочных эффектов, одним из которых является остеопороз. И об этом обязательно следует помнить.

Таким образом, исходя из вышеуказанной взаимосвязи гормонов ЩЖ и остеопороза, пациентам с нарушениями функции ЩЖ рекомендован прием препаратов кальция с целью нивелировать, предупредить или скорректировать уже развившийся остеопороз. При этом очень важно помнить, что на этапе всасывания L-тироксин и препараты кальция чисто механически могут взаимодействовать. Поэтому пациентов обязательно следует предупредить о том, что между приемом L-тироксина и кальцийсодержащего препарата должно пройти не менее 30-40 минут. При одновременном же их приеме L-тироксин буде хуже всасываться и эффект заместительной супрессивной терапии достигнут не будет. На практике такие ситуации возникают, к сожалению, достаточно часто.

\section{ЛИТЕРATУPA/REFERENCES \\ 1. Williams, G.R., Bassett, J.H.D. \\ “Thyroid diseases and bone health." J Endocrinol Invest 41 (2018): 99-109. ๑}



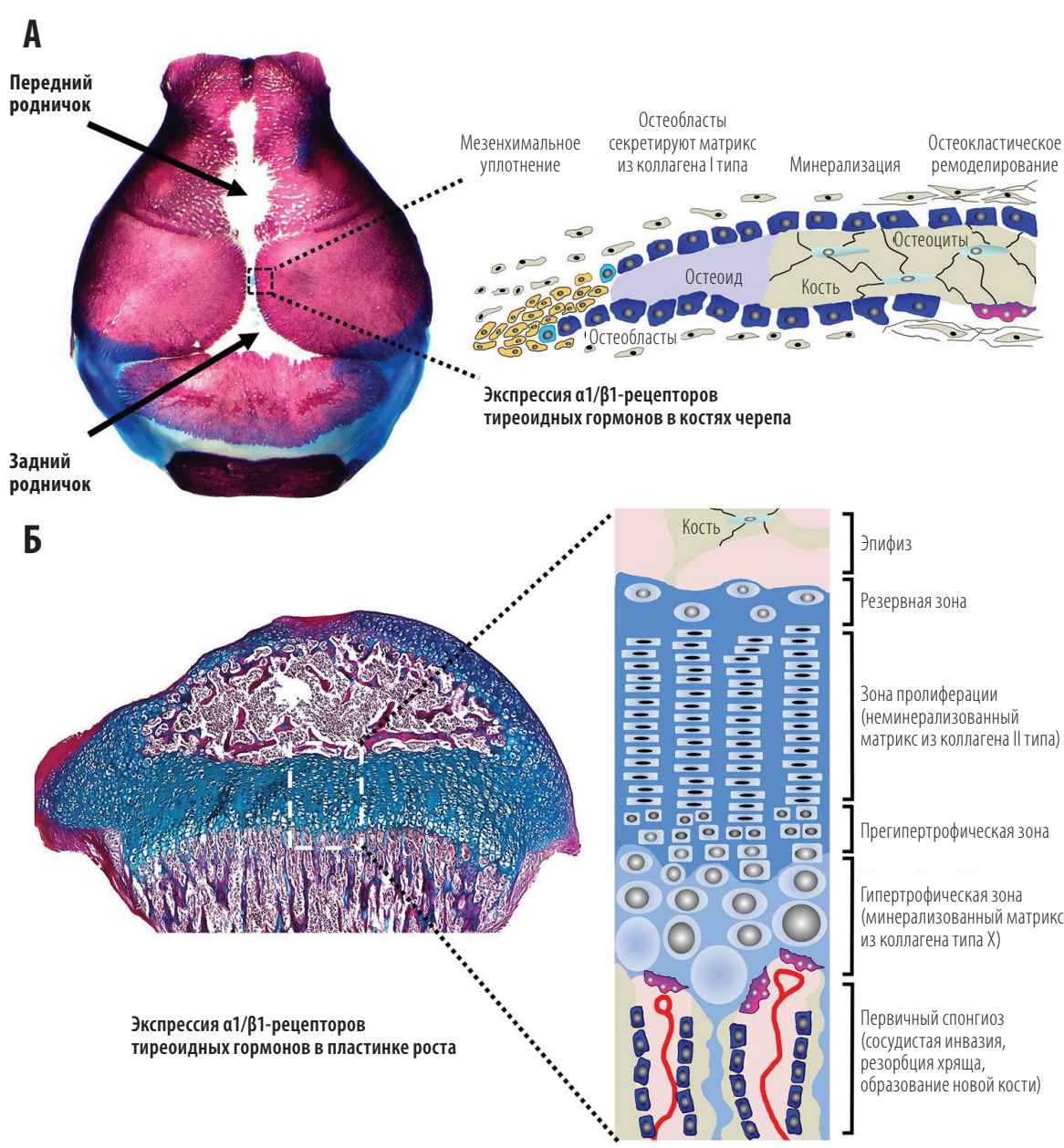

Рисунок 1. Внутримембранное и эндохондральное окостенение [1]

А. Свод черепа, окрашенный ализариновым красным (кость) и альциановым синим (хрящ) с 1-го дня после рождения, стрелками указаны передний и задний роднички, а также швы. Схематическое изображение внутримембранного образования костей черепа.

В. Сечение проксимального отдела большеберцовой кости на 21-й день после рождения, альциановым синим окрашен хрящ, костный матрикс окрашен красным по ван Гизону. Схематическое изображение эндохондрального окостенения, показывающее пролиферацию, дифференцировку и апоптоз хондроцитов в ростовой пластинке.

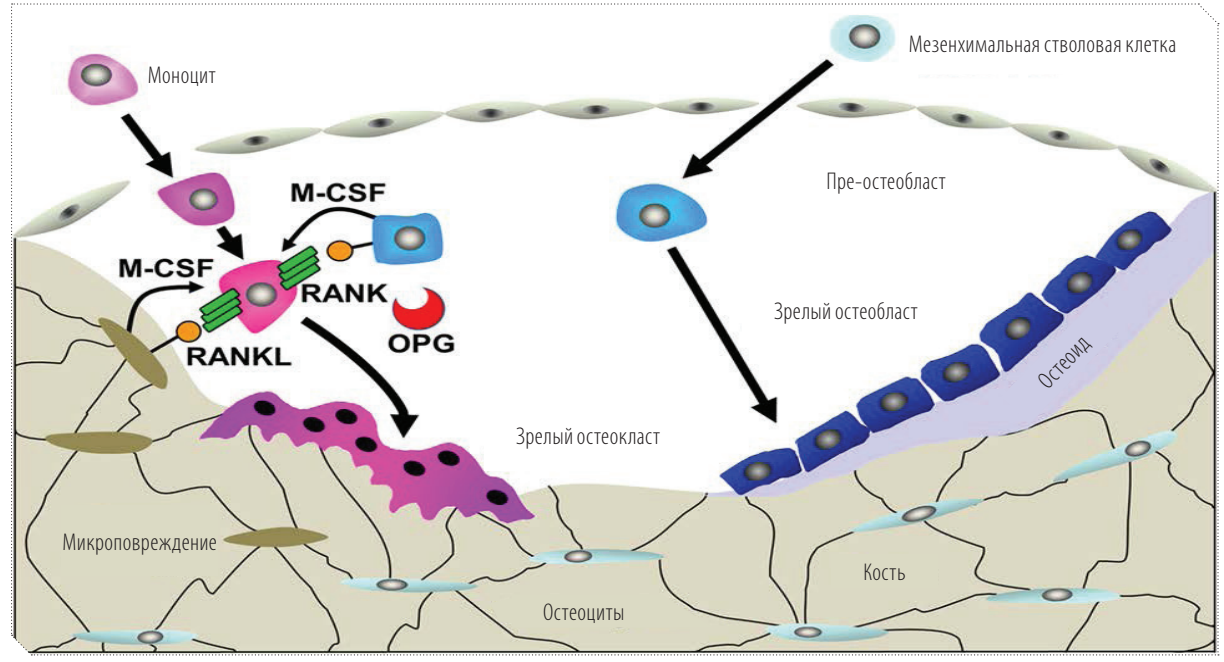

Рисунок 2. Цикл ремоделирования кости [1]

Цикл ремоделирования кости обеспечивается остеоцитами, структурными элементами кости. Ремоделирование кости начинается с изменения механической нагрузки, структурного микроповреждения или воздействия системных или паракринных факторов. Предшественники моноцитов/макрофагов дифференцируются в зрелые остеокласты и резорбируют костную ткань. Дифференцировка индуцируется макрофагальным колониестимулирующим фактором (M-CSF) и активатором рецептора лиганда NFkB (RANKL), а ингибируется остеопротегерином (OPG). После резорбции образуются предшественники остеобластов, синтезируют остеоидный матрикс и регулируют его минерализацию для формирования новой костной ткани, таким образом восстанавливая дефект.

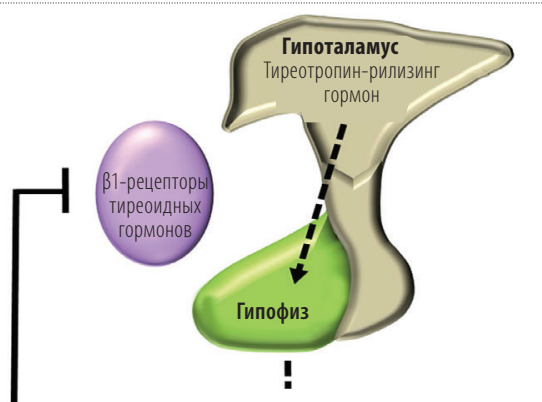

Тиреостимулирующий гормон

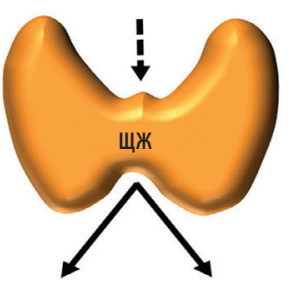

T4

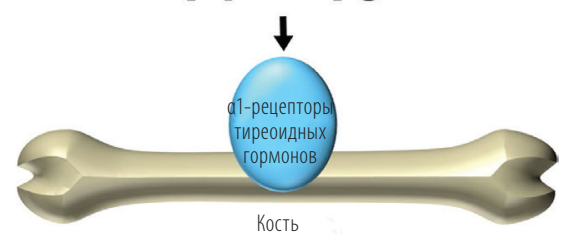

Рисунок 3. Ось гипоталамус-гипофиз-ЩЖ [1] ЩЖ секретирует прогормон Т и и активный гормон $\mathrm{T}_{3^{\prime}}$ концентрации гормонов в крови регулируются классической эндокринной отрицательной обратной связью, которая поддерживает обратную физиологическую связь между тиреостимулирующим гормоном и $\mathrm{T}_{4}$ и $\mathrm{T}_{3}$.

\section{T4}

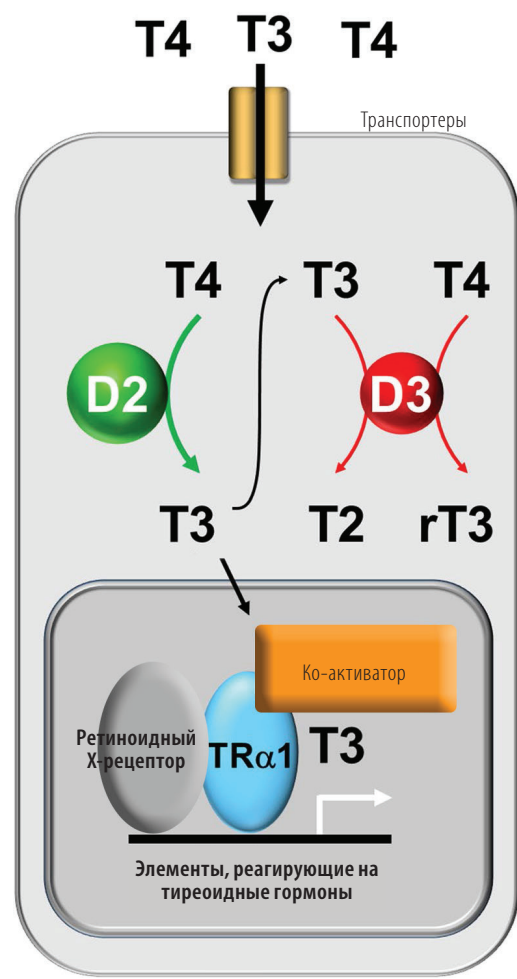

Рисунок 4. Действие гормонов ЩЖ в клетках костной ткани [1]

Гормоны ЩЖ попадают в клетки-мишени $\mathrm{T}_{3}$ через

специфические мембранные транспортеры и регулируют активность дейодиназ типов 2 и 3 ( $D_{2}$ и $\left.D_{3}\right)$, что

обеспечивает оптимальную доступность внутриклеточного Т. Это приводит к смещению ко-репрессора и связыванию ко-активатора и, таким образом, к физиологической транскрипционной активности а1-рецепторов тиреоидных гормонов (TRa1). 